\title{
Métodos não farmacológicos para alívio da dor durante o trabalho de parto: revisão sistemática
}

\author{
Non-pharmacological methods for pain relief during labor: systematic review
}

Métodos no farmacológicos para aliviar el dolor durante el part: revisión sistemática

Ana Cláudia Costa Pereira ${ }^{1 *}$, Ana Luiza Melo Lima Costa ${ }^{1}$, Alice Brant Costa ${ }^{1}$, Bettina Geber ${ }^{1}$, Bruna Ferreira Alkmim¹, Giulia Costa Val Camaranoㄹ, Rafaela Resende da Glória², Taruany Melo Nogueira', Vitória Alagia Ripari ${ }^{1}$, Adriel Gustavo Lopes ${ }^{1}$.

\section{RESUMO}

Objetivo: Avaliar por meio de uma revisão a importância dos métodos não farmacológicos para a construção de uma melhor vivência do trabalho de parto. Métodos: Revisão sistemática realizada nas bases de dados MEDLINE/PubMed, LILACS e SciELO, com o uso dos descritores "Terapias Complementares" e "Dor do Parto", juntamente com o operador lógico de pesquisa "AND". Incluiu-se artigos em inglês, espanhol e português com o recorte temporal de 2016 a 2020. Resultados: Foram selecionados 19 artigos de ensaios clínicos randomizados. Avaliou-se as seguintes técnicas complementares: acupressão, auriculoterapia, aromaterapia, bola suíça, exercício respiratório, yoga, terapia floral, massagem, métodos térmicos e banho de aspersão como métodos não farmacológicos para o controle da dor durante o trabalho de parto. Considerações Finais: As terapias complementares são importantes aliadas no controle da dor, na promoção de uma sensação de satisfação e no fornecimento de um suporte para as parturientes. O uso destes meios não farmacológicos pode viabilizar uma melhor vivência do trabalho de parto, proporcionando um parto mais humanizado ao colocar a mulher como protagonista.

Palavras-chave: Dor do parto, Terapias complementares, Parto humanizado.

\section{ABSTRACT}

Objective: To evaluate through a review the importance of non-pharmacological methods for building a better labor experience. Methods: Systematic review carried out in the MEDLINE/Pubmed, LILACS and SciELO databases, using the descriptors "Complementary therapies" and "Labor pain", with the logical search operator "AND". Articles in English, Spanish and Portuguese were included within the period of 2016 to 2020. Results: 19 articles from randomized clinical trials were selected. The following complementary therapies were evaluated: acupressure, auriculotherapy, aromatherapy, swiss ball, breathing exercises, yoga, flower therapy, massage, thermal methods and spray bath as non-pharmacological methods for pain control during labor. Final considerations: Complementary therapies are important allies in controlling pain, promoting a feeling of satisfaction and providing support for parturient women. The use of these non-pharmacological means can enable a better experience of labor, providing a more humanized delivery by placing the woman as the protagonist.

Keywords: Labor pain, Complementary therapies, Humanizing delivery.

\section{RESUMEN}

Objetivo: Evaluar a través de una revisión la importancia de los métodos no farmacológicos para construir una mejor experiencia laboral. Métodos: Revisión sistemática realizada en las bases de datos MEDLINE/PubMed, LILACS y SCIELO, utilizando los descriptores "Terapias complementarias" y "Dor de parto", con el operador de búsqueda lógica "Y". Se incluyeron artículos en inglés, español y portugués con el marco temporal de 2016 a 2020. Resultados: Se seleccionaron 19 artículos de ensayos clínicos aleatorios. Se evaluaron las siguientes técnicas complementarias: acupresión, auriculoterapia, aromaterapia, pelota suiza, ejercicios de respiración, yoga, terapia de flores, masajes, métodos térmicos y baños de spray como métodos no farmacológicos para el control del dolor durante el trabajo de parto. Consideraciones finales: Las terapias complementarias son aliados importantes para controlar el dolor, promover un sentimiento de satisfacción y brindar apoyo a las mujeres parturientas. El uso de estos medios no farmacológicos puede permitir una mejor experiencia del parto, proporcionando un parto más humanizado al colocar a la mujer como protagonista.

Palabras clave: Dolor de parto, Terapias complementarias, Parto humanizado.

${ }^{1}$ Pontifícia Universidade Católica de Minas Gerais, Betim - MG. *E-mail: anaclaudiacpereira@gmail.com

${ }^{2}$ Faculdade Ciências Médicas de Minas Gerais, Belo Horizonte - MG. 


\section{INTRODUÇÃO}

A dor no trabalho de parto está entre as dores mais intensas conhecidas (SURUCU SG, et al., 2018). A percepção da parturiente sobre a dor depende não somente de fatores biológicos, como a intensidade e duração das contrações uterinas, a condição física da gestante, a nuliparidade e a pressão exercida pelo feto nas estruturas pélvicas no período expulsivo, mas também de fatores psíquicos, sociais e culturais, ou seja, das experiências prévias da parturiente, das expectativas geradas durante a gestação e da ausência do acompanhante (MAFETONI RR, et al., 2019).

Diante dessa série de eventos que se interagem durante o processo do parto é comum observar que as pacientes experimentem a sensação de ansiedade. Contudo, como demonstrado por Boaviagem A, et al. (2016), os altos níveis de ansiedade nesse período contribuem para o aumento da dor física, uma vez que pode provocar espasmos e maior contração da musculatura pélvica e lombar.

Com isso, pode-se observar um sistema de retroalimentação, no qual ansiedade, dor, estímulos internos e estímulos externos se integram e resultam na experiência pessoal e única de cada gestante durante o trabalho de parto. Desse modo, a abordagem psicológica da gestante, de seus medos, inseguranças e sentimentos em relação ao trabalho de parto, desde o início do acompanhamento pré-natal, pode exercer influência benéfica na experiência da gestação e do parto.

Conforme Ozgoli G, et al. (2016), a abordagem médica convencional para o tratamento da dor no trabalho de parto e parto passou a depender cada vez mais dos métodos farmacológicos, dentre eles a analgesia peridural. Apesar de sua eficácia no alívio da dor, ela pode resultar em trabalho de parto prolongado, causar uma maior necessidade de partos vaginais instrumentais e cesarianas, risco de hipotensão, bloqueio motor, febre, retenção urinária e problemas de amamentação (TANVISUT R, et al., 2018). Devido a estes efeitos colaterais, o uso de métodos não farmacológicos tem sido muito procurado pelas parturientes em busca de uma nova medida para o alívio da dor (TAAVONI S, et al., 2016).

De acordo com Silva CA e Lara SRG (2018), prestar uma assistência de qualidade, em que o processo de parturição seja valorizado como fisiológico e seja realizado com o uso adequado de tecnologia, como os métodos não farmacológicos para o alívio da dor, é um dos pilares do processo gravídico-puerperal. Segundo Sucuru SG, et al. (2018), estes métodos não precisam de auxílio médico e são inofensivos para mãe e para o bebê. Eles não diminuem o tempo do trabalho de parto e afetam positivamente a sua progressão. Além disso, eles não causam efeitos colaterais ou alergias. Dentre as práticas recomendadas, destacam-se: acupuntura, auriculoterapia, hidroterapia, aromaterapia, bola suíça, exercícios respiratórios e massagem.

Apesar de não ser necessário o auxílio médico durante a realização desses métodos, é sempre válido e de grande importância a presença de doulas, fisioterapeutas e enfermeiros que ajudem a gestante durante 0 seu trabalho de parto, visto que essa ajuda, de acordo com Akkoz S e Karaduman, S (2019), aumenta o vínculo com a mulher e a deixa mais à vontade nesse momento difícil.

Somado a isso, a presença de dor não implica necessariamente uma experiência negativa no parto, as mulheres podem ter dor coexistindo com satisfação, prazer e empoderamento (AKKOZ S e KARADUMAN S, 2019). Dessa maneira, partindo-se do que é observado nas maternidades e levando em consideração a excessiva utilização destes tratamentos farmacológicos no trabalho de parto, a presente pesquisa reúne vários exemplos de métodos alternativos de tratamentos não farmacológicos no intuito de avaliar a importância desses métodos para a construção de uma melhor vivência do trabalho de parto, a partir da investigação de como essas terapias atuam sobre o alívio da dor e a redução da ansiedade durante o trabalho de parto

Diante disso, o objetivo desse trabalho é avaliar a importância dos métodos não farmacológicos para a construção de uma melhor vivência do trabalho de parto, buscando alívio da dor e diminuição da ansiedade e do estresse. As principais hipóteses são o uso de meios não farmacológicos como importantes coadjuvantes para o alívio da dor durante o parto e a utilização dessas terapias complementares na melhora da ansiedade e do estresse materno na vivência do trabalho de parto, propiciando um parto mais humanizado. 


\section{MÉTODOS}

Trata-se de um estudo de revisão sistemática pautado na seguinte pergunta norteadora: "Existem evidências científicas de que os meios não-farmacológicos são eficazes para auxiliar no alívio da dor durante o trabalho de parto?".

A realização da busca se deu nas seguintes bases de dados: Medical Literature and Retrivial System onLine (MEDLINE/PubMed), Literatura Latino-americana e do Caribe em Ciências da Saúde (LILACS) e Scientific Electronic Library Online (SciELO), utilizando-se os descritores "Terapias Complementares" e "Dor do Parto", selecionados previamente no Descritores em Ciências da Saúde (DeCs). Durante o processo de busca, foi usado o operador lógico de pesquisa "AND".

Como critérios de inclusão foram aplicados: ensaios clínicos randomizados, realizados com humanos, nos idiomas inglês, português e espanhol com recorte temporal entre os anos de 2016 a 2020, que se adequam à pergunta norteadora da pesquisa. Foram excluídos os artigos não acessíveis e os que não estavam atrelados ao objetivo da pesquisa, por meio da leitura do título e do resumo. A busca bibliográfica foi realizada durante o mês de Abril de 2020.

Diante do processo de seleção dos artigos foram aplicadas as etapas de identificação, triagem e elegibilidade, ilustradas no fluxograma a seguir (Figura 1). Por fim, foi realizada a avaliação crítica dos estudos encontrados para posterior aplicação dos resultados e síntese das evidências.

Figura 1 - Fluxograma do processo de seleção: identificação, triagem e elegibilidade.
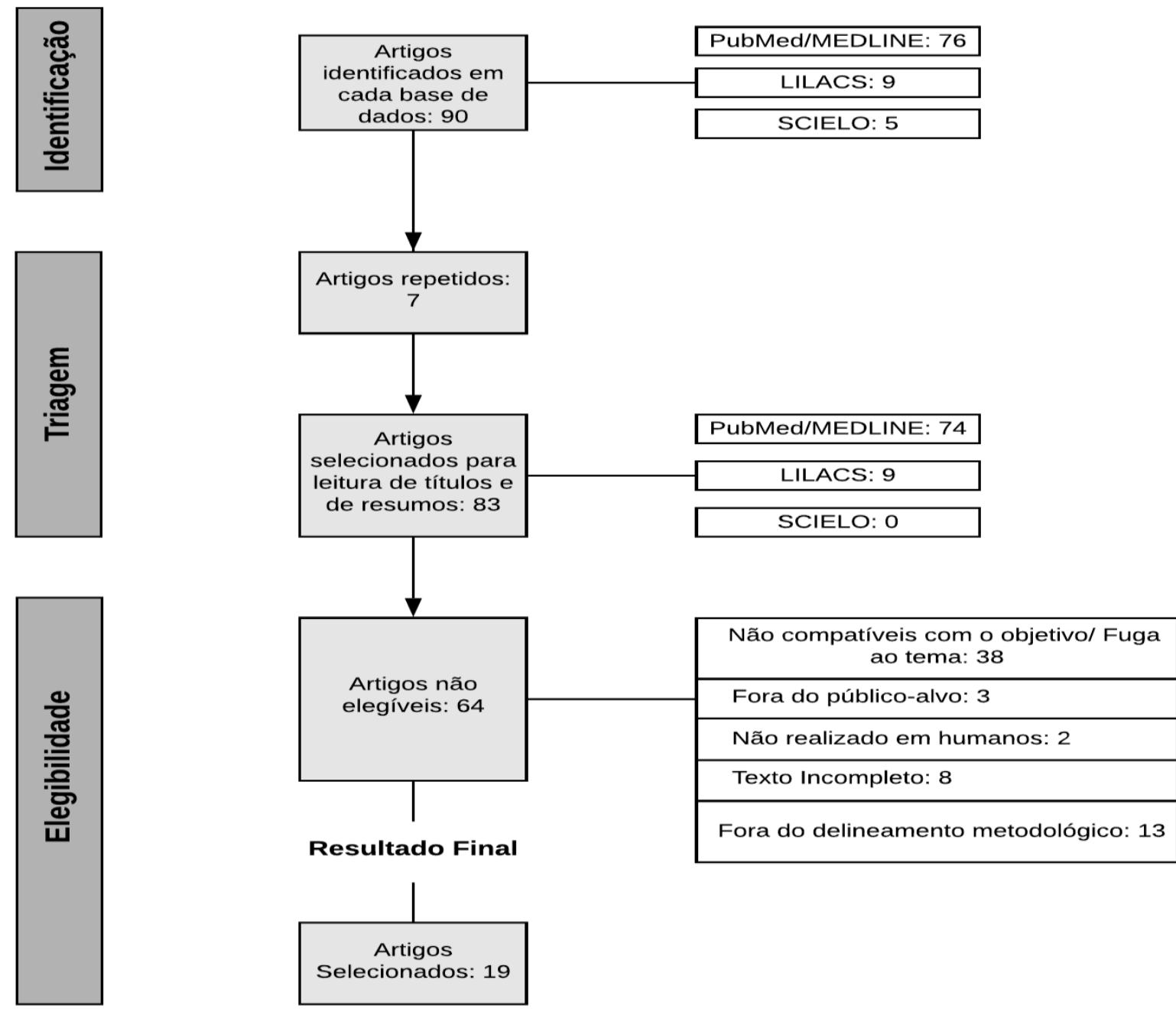

Artigos

Selecionados: 19

Fonte: Pereira ACC, et al., 2020. 


\section{RESULTADOS}

Nesta revisão analisou-se 19 artigos de estudos clínicos randomizados. Foram avaliadas as seguintes técnicas complementares: acupressão, auriculoterapia, aromaterapia, bola suíça, exercício respiratório, massagem, métodos térmicos e banho de aspersão como métodos não farmacológicos para o controle da dor durante o trabalho de parto.

Em relação às temáticas discutidas, encontrou-se: cinco artigos de acupressão, publicados no período de 2016 a 2019; três artigos de aromaterapia, entre 2016 e 2018; dois artigos de exercícios respiratórios de 2017; dois artigos de massagem de 2017 e de 2019; dois artigos que incluem conjuntamente a bola suíça e termoterapia/banho quente de aspersão em 2016 e em 2019; dois artigos de auriculoterapia de 2016 e de 2019; um artigo de musicoterapia de 2018; um artigo de yoga de 2017 e um artigo de terapia floral de 2020.

Para a avaliação da intensidade da dor durante o trabalho de parto foi utilizada de modo geral, como instrumento de medida de dor nos estudos, a Escala Visual Analógica (EVA), ferramenta importante para aferir também a evolução das queixas álgicas com o passar do tempo. Os principais dados obtidos dos referenciais teóricos podem ser analisados no Quadro 1. 


\section{Revista Eletrônica Acervo Saúde / Electronic Journal Collection Health ISSN 2178-2091}

Quadro 1 - Principais dados dos referenciais teóricos utilizados nesta pesquisa

\begin{tabular}{|c|c|c|c|c|}
\hline Autor/Ano & Título & $\begin{array}{c}\text { Terapia } \\
\text { Complementar }\end{array}$ & Objetivos & Resultados \\
\hline $\begin{array}{c}\text { Tanvisut R, et al. } \\
(2018)\end{array}$ & $\begin{array}{l}\text { Efficacy of aromatherapy for reducing } \\
\text { pain during labor: a randomized } \\
\text { controlled trial }\end{array}$ & Aromaterapia & $\begin{array}{l}\text { Determinar a eficácia da aromaterapia } \\
\text { na redução da dor durante o trabalho } \\
\text { de parto. }\end{array}$ & $\begin{array}{l}\text { O escore mediano de dor da fase ativa latente e precoce foi } \\
\text { menor no grupo de aromaterapia, } 5 \text { vs } 6 \text { e } 7 \text { vs } 8 \text {, } \\
\text { respectivamente. Já os escores de dor tardia na fase ativa } \\
\text { não foram significativamente diferentes. }\end{array}$ \\
\hline $\begin{array}{l}\text { Yazdkhasti M e } \\
\text { Pirak A (2016) }\end{array}$ & $\begin{array}{l}\text { The effect of aromatherapy with } \\
\text { lavender essence on severity of labor } \\
\text { pain and duration of labor in } \\
\text { primiparous women }\end{array}$ & Aromaterapia & $\begin{array}{l}\text { Investigar o efeito da inalação da } \\
\text { essência de lavanda na severidade da } \\
\text { dor no parto e duração do trabalho de } \\
\text { parto. }\end{array}$ & $\begin{array}{l}\text { Os resultados mostraram que a diferença na dor do parto } \\
\text { antes e após a intervenção em dois grupos foi significativo } \\
(\mathrm{P} 1 / 40 / 001) \text {. Mas não houve diferença na duração média da } \\
\text { fase ativa e no tempo de segunda etapa do trabalho de parto } \\
\text { entre os dois grupos. }\end{array}$ \\
\hline $\begin{array}{l}\text { Hamdamian S, et } \\
\text { al. (2018) }\end{array}$ & $\begin{array}{l}\text { Effects of aromatherapy with Rosa } \\
\text { damascena on nulliparous women's } \\
\text { pain and anxiety of labor during first } \\
\text { stage of labor }\end{array}$ & Aromaterapia & $\begin{array}{l}\text { Esse estudo tem como objetivo avaliar } \\
\text { os efeitos de aromaterapia com Rosa } \\
\text { damascena na dor e ansiedade } \\
\text { durante o primeiro estágio do parto em } \\
\text { mulheres nulíparas. }\end{array}$ & $\begin{array}{l}\text { A aromaterapia com Rosa damascena permitiu níveis de dor } \\
\text { significativamente menores que o grupo controle, durante } \\
\text { cada ponto de avaliação, dilatação cervical de } 4-5,6-7 \text { e } 8 \text { - } \\
10 \mathrm{~cm}(p<0,05) \text {. Os níveis de ansiedade também foram } \\
\text { significantemente menores, avaliado no processo de } \\
\text { dilatação cervical em } 4-7 \text { e } 8-10 \mathrm{~cm}(p<0,05) \text {. }\end{array}$ \\
\hline $\begin{array}{l}\text { Yuksel H, et al. } \\
\qquad(2017)\end{array}$ & $\begin{array}{l}\text { Effectiveness of breathing exercises } \\
\text { during the second stage of labor on } \\
\text { labor pain and duration: a } \\
\text { randomized controlled trial }\end{array}$ & $\begin{array}{l}\text { Exercício } \\
\text { Respiratório }\end{array}$ & $\begin{array}{l}\text { Determinar se os exercícios } \\
\text { respiratórios para mulheres grávidas } \\
\text { durante o segundo estágio do trabalho } \\
\text { de parto têm efeitos benéficos na dor } \\
\text { materna, na duração do trabalho de } \\
\text { parto e nas pontuações de Aparência, } \\
\text { Pulso, Careta, Atividade e Respiração } \\
\text { (APGAR) no primeiro minuto. }\end{array}$ & $\begin{array}{l}\text { Com base neste estudo, exercícios respiratórios com } \\
\text { inspiração e expiração profundas em gestantes são eficazes } \\
\text { em reduzir a percepção da dor do parto e encurtar a duração } \\
\text { do segundo estágio de entrega. }\end{array}$ \\
\hline $\begin{array}{c}\text { Boaviagem A, et } \\
\text { al. (2017) }\end{array}$ & $\begin{array}{l}\text { The effectiveness of breathing } \\
\text { patterns to control maternal anxiety } \\
\text { during the first period of labor: A } \\
\text { randomized controlled clinical trial }\end{array}$ & $\begin{array}{c}\text { Exercício } \\
\text { Respiratório }\end{array}$ & $\begin{array}{l}\text { Avaliar a eficácia dos padrões } \\
\text { respiratórios durante a fase ativa do } \\
\text { primeiro estágio do trabalho de parto } \\
\text { para a ansiedade materna. }\end{array}$ & $\begin{array}{l}\text { Os resultados do estudo não mostraram diferença } \\
\text { significativa entre os grupos no padrão de eficácia da } \\
\text { respiração com respiração lenta e profunda, suspiro } \\
\text { expiratório e atraso expiratório no controle da ansiedade, } \\
\text { dor, fadiga e satisfação materna durante o primeiro estágio } \\
\text { do trabalho de parto. }\end{array}$ \\
\hline $\begin{array}{l}\text { Mafetoni RR, et al. } \\
\qquad(2019)\end{array}$ & $\begin{array}{l}\text { Efetividade da auriculoterapia sobre } \\
\text { a dor no trabalho de parto: ensaio } \\
\text { clínico randomizado }\end{array}$ & Auriculoterapia & $\begin{array}{l}\text { Avaliar a efetividade da auriculoterapia } \\
\text { sobre a dor na fase ativa do trabalho } \\
\text { de parto. }\end{array}$ & $\begin{array}{l}\text { As médias de intensidade da dor foram parecidas na } \\
\text { admissão (intervenção: } 7,2 \pm 1,6 \text { vs placebo: } 6,9 \pm 2,4 \text { vs } \\
\text { controle: } 7,5 \pm 1,8 ; p \text {-valor }=0,4475 \text { ), porém com } 60 \text { minutos } \\
\text { (intervenção: } 6,8 \pm 1,9 \text { vs placebo: } 7,5 \pm 2,4 \text { vs controle: } \\
8,3 \pm 1,8 ; p=0,0060 \text { ) e } 120 \text { minutos (intervenção: } 7,1 \pm 1,9 \text { vs } \\
\text { placebo: } 8,0 \pm 2,4 \text { vs controle: } 8,8 \pm 1,9 ; p \text {-valor }=0,039 \text { ) houve } \\
\text { aumento significativo nos escores dor entre as parturientes } \\
\text { dos grupos placebo e controle. }\end{array}$ \\
\hline
\end{tabular}

REAS/EJCH | Vol.12(10) | e4448 | DOI: https://doi.org/10.25248/reas.e4448.2020 Página 5 de 11 


\section{Revista Eletrônica Acervo Saúde / Electronic Journal Collection Health ISSN 2178-2091}

\begin{tabular}{|c|c|c|c|c|}
\hline Autor/Ano & Título & $\begin{array}{c}\text { Terapia } \\
\text { Complementar }\end{array}$ & Objetivos & Resultados \\
\hline $\begin{array}{c}\text { Mafetoni RR e } \\
\text { Shimo AKK (2016) }\end{array}$ & $\begin{array}{l}\text { Efeitos da auriculoterapia sobre a dor } \\
\text { do trabalho de parto: ensaio clínico } \\
\text { randomizado }\end{array}$ & Auriculoterapia & $\begin{array}{l}\text { Avaliar os efeitos da auriculoterapia no } \\
\text { controle da dor e seus desfechos na } \\
\text { duração do trabalho de parto. }\end{array}$ & $\begin{array}{l}\text { Não houve significância estatística entre os grupos com } \\
\text { relação à dor; no entanto, as mulheres do grupo de } \\
\text { auriculoterapia, apresentaram menor intensidade e menor } \\
\text { percepção da dor aos } 30,60 \text { e } 120 \text { minutos do tratamento. A } \\
\text { média de duração do trabalho de parto foi menor no grupo } \\
\text { de auriculoterapia. }\end{array}$ \\
\hline $\begin{array}{l}\text { Hamlacđ Y e } \\
\text { Yazici S (2017) }\end{array}$ & $\begin{array}{l}\text { The Effect of Acupressure Applied to } \\
\text { Point LI4 on Perceived Labor Pains }\end{array}$ & Acupressão & $\begin{array}{l}\text { Determinar o efeito de acupressão no } \\
\text { ponto LI4 na percepção da dor do } \\
\text { parto. }\end{array}$ & $\begin{array}{l}\text { Como mostrado no estudo, aplicar acupressão no ponto LI4 } \\
\text { se mostrou efetivo na diminuição da percepção da dor de } \\
\text { parto e diminuição do tempo de parto }(P<0.05) \text {. }\end{array}$ \\
\hline $\begin{array}{l}\text { Ozgoll G, et al. } \\
\qquad(2016)\end{array}$ & $\begin{array}{l}\text { Effect of LI4 and BL32 acupressure } \\
\text { on labor pain and delivery outcome in } \\
\text { the first stage of labor in primiparous } \\
\text { women: A randomized controlled trial }\end{array}$ & Acupressão & $\begin{array}{l}\text { Comparar o efeito da acupressão LI4 e } \\
\text { BL32 entre si e o grupo controle sobre } \\
\text { a dor no parto e os resultados no } \\
\text { parto. }\end{array}$ & $\begin{array}{l}\text { A redução da dor foi significativamente maior nos grupos LI4 } \\
\text { e BL32 em comparação com o controle em todos os } \\
\text { períodos de estudo. Além disso, a acupressão no ponto } \\
\text { BL32 foi superior ao ponto LI4 no alívio da dor na primeira e } \\
\text { segunda, mas não terceira intervenção. }\end{array}$ \\
\hline $\begin{array}{l}\text { Mafetoni RR e } \\
\text { Shimo AKK } \\
(2016)\end{array}$ & $\begin{array}{l}\text { Efeitos da acupressão sobre a dor no } \\
\text { trabalho de parto: ensaio clínico } \\
\text { randomizado }\end{array}$ & Acupressão & $\begin{array}{l}\text { Analisar os efeitos da acupressão no } \\
\text { ponto sanyinjiao sobre a dor na fase } \\
\text { ativa do trabalho de parto, em } \\
\text { gestantes atendidas em maternidade } \\
\text { pública. }\end{array}$ & $\begin{array}{l}\text { As médias de dor pela EAV não foram diferentes nos três } \\
\text { grupos na admissão ( } p \text {-valor }=0,0929) \text {, porém foram menores } \\
\text { no grupo de acupressão imediatamente após }(p- \\
\text { valor }=<0,0001 \text { ) e com } 1 \mathrm{~h} \text { do tratamento ( } p \text {-valor }=0,0001) \text { ao } \\
\text { se comparar com placebo e controle. }\end{array}$ \\
\hline $\begin{array}{c}\text { Turkmen H e } \\
\text { Turfan EÇ (2019) }\end{array}$ & $\begin{array}{l}\text { The Effect of Acupressureon Labor } \\
\text { Pain and the Duration of Labor When } \\
\text { Applied to the SP6 Point: } \\
\text { Randomized Clinical Trial }\end{array}$ & Acupressão & $\begin{array}{l}\text { Determinar os efeitos da acupressão } \\
\text { no ponto SP6 durante o primeiro } \\
\text { estágio do trabalho parto na dor e em } \\
\text { sua duração. }\end{array}$ & $\begin{array}{l}\text { O nível de dor percebida no estágio ativo no grupo } \\
\text { experimental foi menor que o grupo controle (dor no } \\
\text { trabalho: } 7,17+0,89 \text { vs. } 7,66+0,71, p-0,002) \text {. A duração } \\
\text { média da primeira etapa do trabalho de parto no grupo } \\
\text { experimental foi mais curta que no grupo controle }(4,88+ \\
0,85 \mathrm{~h} \text { vs. } 5,56 \pm 0,66, p=0,001 \text { ). Constatou-se que mulheres } \\
\text { grávidas do grupo experimental recomendariam esse } \\
\text { método a outras mulheres grávidas (qui-quadrado }-5,711 \text {, } \\
p=0,017 \text { ). }\end{array}$ \\
\hline $\begin{array}{l}\text { Yildirim E, et al. } \\
\qquad(2018)\end{array}$ & $\begin{array}{l}\text { The effect of ice pressure applied on } \\
\text { large intestinal } 4 \text { on the labor pain } \\
\text { and labor process }\end{array}$ & Acupressão & $\begin{array}{l}\text { Estudo experimental controlado, que } \\
\text { objetiva identificar o efeito da } \\
\text { massagem de gelo com pequena } \\
\text { duração ( } 40 \text { minutos) e longa duração } \\
\text { ( } 80 \text { minutos) aplicadas no ponto LI4 de } \\
\text { acupressão durante o processo do } \\
\text { parto e a percepção da dor no parto. }\end{array}$ & $\begin{array}{l}\text { O tempo e magnitude das contrações em } 80 \text { minutos após a } \\
\text { intervenção foi significantemente maior no grupo que sofreu } \\
\text { a intervenção }(p<0,05), \text { o apagamento do colo foi } \\
\text { estatisticamente maior }(p<0,05) \text { e houve redução importante } \\
\text { da duração do parto nesse grupo ( }(p<0,001) \text {. Mulheres do } \\
\text { grupo da acupressão necessitaram de menos episiotomias } \\
\text { que o grupo controle }(p=0,032) \text { e obtiveram redução positiva } \\
\text { na percepção de dor }(p=0,001) \text {. }\end{array}$ \\
\hline $\begin{array}{l}\text { Cavalcanti ACV, et } \\
\text { al. (2019) }\end{array}$ & $\begin{array}{l}\text { Terapias complementares no } \\
\text { trabalho de parto: ensaio clínico } \\
\text { randomizado }\end{array}$ & $\begin{array}{l}\text { Banho quente de } \\
\text { chuveiro e } \\
\text { exercício perineal } \\
\text { com bola suíça }\end{array}$ & $\begin{array}{l}\text { Avaliar o efeito do banho quente de } \\
\text { chuveiro e do exercício perineal com } \\
\text { bola suíça, isolados e de forma } \\
\text { combinada, sobre a percepção da dor, } \\
\text { ansiedade e progressão do trabalho } \\
\text { de parto. }\end{array}$ & $\begin{array}{l}\text { Houve aumento no escore de dor e redução da ansiedade } \\
\text { em todos os grupos, sobretudo quando utilizaram banho de } \\
\text { chuveiro. A dilatação cervical, aumentou em todos os grupos } \\
\text { de intervenção }(p<, 001) \text { bem como o número de contrações } \\
\text { uterinas, principalmente quem utilizou banho e bola } \\
\text { associados, como também mostrou menor duração do } \\
\text { tempo de trabalho de parto. }\end{array}$ \\
\hline
\end{tabular}

REAS/EJCH | Vol.12(10) | e4448 | DOI: https://doi.org/10.25248/reas.e4448.2020

Página 6 de 11 


\section{Revista Eletrônica Acervo Saúde / Electronic Journal Collection Health ISSN 2178-2091}

\begin{tabular}{|c|c|c|c|c|}
\hline Autor/Ano & Título & $\begin{array}{c}\text { Terapia } \\
\text { Complementar }\end{array}$ & Objetivos & Resultados \\
\hline $\begin{array}{l}\text { Taavoni S,et al. } \\
\qquad(2016)\end{array}$ & $\begin{array}{l}\text { Birth ball or heat therapy? A } \\
\text { randomized controlled trial to } \\
\text { compare the effectiveness of birth } \\
\text { ball usage with sacrum-perineal heat } \\
\text { therapy in labor pain management }\end{array}$ & $\begin{array}{l}\text { Bola de parto e } \\
\text { terapia pelo calor }\end{array}$ & $\begin{array}{l}\text { Comparar os efeitos de dois métodos } \\
\text { não-farmacológicos, bola de parto e } \\
\text { terapia de calor, para o alívio da dor no } \\
\text { parto e sua duração durante a fase } \\
\text { ativa do parto fisiológico. }\end{array}$ & $\begin{array}{l}\text { O escore médio de gravidade da dor no grupo de } \\
\text { termoterapia foi inferior ao do grupo controle aos } 60 \text { e } 90 \\
\text { minutos após a intervenção }(p<0,05) \text {. Além disso, houve } \\
\text { diferenças significativas entre os escores de dor no parto no } \\
\text { grupo da bola depois dos três tempos investigados em } \\
\text { comparação ao grupo controle. }\end{array}$ \\
\hline $\begin{array}{l}\text { Surucu SG, et al. } \\
\qquad(2018)\end{array}$ & $\begin{array}{l}\text { The Effect of Music on Pain and } \\
\text { Anxiety of Women During Labour on } \\
\text { First Time Pregnancy: A Study From } \\
\text { Turkey }\end{array}$ & Musicoterapia & $\begin{array}{l}\text { Analisar o efeito da música na dor e } \\
\text { ansiedade sentidas pelas mulheres em } \\
\text { trabalho de parto durante a primeira } \\
\text { gravidez. }\end{array}$ & $\begin{array}{l}\text { Observou-se que após a primeira hora as mulheres } \\
\text { indicaram que a dor era estatisticamente menor no grupo } \\
\text { experimental. Os escores de ansiedade das mulheres em } \\
\text { trabalho de parto foram semelhantes nos grupos } \\
\text { experimental e controle. Após a prática, os escores médios } \\
\text { de ansiedade ficaram mais baixos em favor do grupo } \\
\text { experimental e a correlação foi estatisticamente significante. }\end{array}$ \\
\hline $\begin{array}{l}\text { Erdogan SU, et al. } \\
\qquad(2017)\end{array}$ & $\begin{array}{l}\text { Effects of low back massage on } \\
\text { perceived birth pain and satisfaction }\end{array}$ & Massagem lombar & $\begin{array}{l}\text { Avaliar o efeito da massagem lombar } \\
\text { na percepção da dor e do parto. }\end{array}$ & $\begin{array}{l}\text { O primeiro escore médio da escala analógica visual de dor } \\
\text { (EVA) foi de } 5,2 \pm 0,9 \text { e } 7,3 \pm 1,3 \text { para os grupos massagem } \\
\text { e controle, respectivamente. O segundo escore da EVA foi } \\
\text { encontrado em } 6,6 \pm 1,6 \text { no grupo massagem e } 8,8 \pm 1,0 \text { no } \\
\text { grupo controle. O terceiro escore da EVA foi } \\
\text { significativamente maior no grupo controle do que no grupo } \\
\text { massagem durante a terceira avaliação }(9,2 \pm 2,4 \text { vs } 6,7 \pm \\
2,7)(p<0,05) \text {. }\end{array}$ \\
\hline $\begin{array}{l}\text { Akkoz S e } \\
\text { Karaduma NS } \\
\quad(2019)\end{array}$ & $\begin{array}{l}\text { The effect of sacral massage on labor } \\
\text { pain and anxiety: A randomized } \\
\text { controlled trial }\end{array}$ & $\begin{array}{l}\text { Massagem } \\
\text { sacral }\end{array}$ & $\begin{array}{l}\text { Determinar o efeito da massagem } \\
\text { sacral na dor e ansiedade no trabalho } \\
\text { de parto. }\end{array}$ & $\begin{array}{l}\text { As médias do EVA da fase latente }(3,57 \pm 1,43) \text {, as médias } \\
\text { do EVA da fase ativa }(7,03 \pm 1,5) \text { e as médias do EVA da } \\
\text { fase de transição }(8,83 \pm 1,78) \text { do grupo experimental foram } \\
\text { estatisticamente significativamente menores do que as do } \\
\text { grupo controle }(P<0.05) \text {. }\end{array}$ \\
\hline $\begin{array}{l}\text { Jahdi F, et al. } \\
\qquad(2017)\end{array}$ & $\begin{array}{l}\text { Yoga during pregnancy: The effects } \\
\text { on labor pain and delivery outcomes } \\
\text { (A randomized controlled trial) }\end{array}$ & Yoga & $\begin{array}{l}\text { Investigar os efeitos de um programa } \\
\text { de yoga pré-natal na dor percebida do } \\
\text { parto materno e nos resultados do } \\
\text { parto. }\end{array}$ & $\begin{array}{l}\text { Os participantes do grupo controle relataram maior } \\
\text { intensidade de dor em comparação ao grupo experimental } \\
(\mathrm{P}<0.05) \text {. Mães do grupo de intervenção pré-natal que } \\
\text { completaram a aula de ioga exigiram uma frequência } \\
\text { reduzida de indução do parto em comparação com o grupo } \\
\text { controle. }\end{array}$ \\
\hline $\begin{array}{c}\text { Lara SRG, et al. } \\
(2020)\end{array}$ & $\begin{array}{l}\text { Vivência de mulheres em trabalho de } \\
\text { parto com o uso de essências florais }\end{array}$ & Terapia Floral & $\begin{array}{l}\text { Descrever a vivência de mulheres } \\
\text { submetidas ao uso de essências } \\
\text { florais como terapia não farmacológica } \\
\text { para o alívio da dor e ansiedade } \\
\text { durante o trabalho de parto. }\end{array}$ & $\begin{array}{l}\text { Constatou-se que os efeitos da terapia floral, atuaram em } \\
\text { sinergia, na redução dos sintomas de estresse-medo-tensão, } \\
\text { além do aumento do bem-estar emocional } \\
\text { proporcionando às parturientes a oportunidade de } \\
\text { protagonizar o seu próprio trabalho de parto e parto. }\end{array}$ \\
\hline
\end{tabular}

Fonte: Pereira ACC, et al., 2020.

REAS/EJCH | Vol.12(10) | e4448 | DOI: https://doi.org/10.25248/reas.e4448.2020 Página 7 de 11 


\section{DISCUSSÃO}

Como já exposto, o trabalho em questão se dedicou à pesquisa de metodologias não farmacológicas para o alívio da dor e redução da ansiedade durante o trabalho de parto. Com isso, os achados da presente pesquisa serão discutidos a seguir, expondo as principais práticas complementares encontradas e quais os efeitos de cada uma sobre o processo do parto.

A aromaterapia consiste na utilização de óleos essenciais concentrados ou essências destiladas de plantas com propriedades terapêuticas, podendo ser ministrada de diferentes maneiras: pela pele por meio de massagens, pela via oral, por meio de compressas e banhos (TANVISUT R, et al., 2018). No entanto, a forma mais utilizada é a inalação. Segundo Yazdkhasti M e Pirak A (2016), acredita-se que esse método é capaz de agir no alívio da dor, da fadiga, da asma, da depressão, da insônia e da ansiedade.

Sendo assim, aplicada durante o trabalho de parto, a aromaterapia tem potencial benéfico no alívio da dor e da ansiedade, muito comuns nesse momento, proporcionando uma experiência mais satisfatória para a mulher. Além disso, de acordo com Hamdamian S, et al. (2018), situações estressantes podem prolongar o trabalho de parto e aumentar o risco de complicações maternas e fetais devido a redução das contrações uterinas, corroborando com a hipótese de que a ação relaxante da aromaterapia é benéfica.

No que diz respeito aos momentos adequados para a aplicação desse método, o estudo de Tanvisut $\mathrm{R}$, et al. (2018) afirma que a maior eficiência se dá na fase latente e no início da fase ativa do trabalho de parto. Na fase ativa avançada, quando a dor se torna mais intensa, a eficácia é reduzida. Por fim, é possível concluir que a aromaterapia é uma alternativa segura, que não traz efeitos colaterais maternos ou fetais e de baixo custo para o alívio da dor. Em adição a isso, podemos destacar sua eficácia na diminuição do número de cesarianas eletivas, já que o medo da dor do parto vaginal é o maior motivo pelo qual as mulheres fazem essa opção (YAZDKHASTI M e PIRAK A, 2016).

Outro método não farmacológico utilizado na tentativa de aliviar as dores do trabalho de parto é o uso de essências florais, que consiste na administração da solução por meio de um conta-gotas. De acordo com Lara, et al. (2020), essa terapia é capaz de afetar os aspectos mentais e emocionais das pacientes, promovendo a restauração de um estado positivo. O bem estar emocional afeta diretamente o bem estar físico devido à ação do sistema nervoso central, do sistema imunológico e do sistema endócrino. Esse método se mostrou bastante eficaz na redução da ansiedade, do estresse e na promoção do relaxamento e do alívio da dor.

Há, na medicina tradicional chinesa, técnicas que auxiliam no controle da dor a partir da estimulação, por pressão, de pontos específicos do corpo humano, tais como a acupressão e a auriculoterapia. Essas duas modalidades de terapia complementar estão sendo amplamente estudadas no contexto da ginecologia e da obstetrícia, mostrando-se eficazes e, com isso, indicadas durante o trabalho de parto com os fins de reduzir a dor e de induzir o parto, bem como no processo de amadurecimento cervical e de ruptura prematura das membranas (MAFETONI RR, et al., 2019; MAFETONI RR e SHIMO AKK, 2016).

A auriculoterapia é uma técnica que consiste em posicionar microesferas de cristais polidos ou agulhas de acupuntura em pontos específicos do pavilhão auditivo e realizar uma pressão sobre eles, de forma a estimular pequenos nervos que, por sua vez, conduzirão o estímulo à medula espinhal e desencadearão uma resposta de liberação de opióides endógenos, provocando alívio da dor.

Assim como mostrado por Mafetoni RR, et al. (2019), o uso da auriculoterapia foi benéfico para as parturientes que receberam estimulação dos pontos correspondentes a estruturas envoltas no trabalho de parto quando comparado em relação àquelas que ou receberam uma terapia placebo - com o uso de microesferas de vidro que, quando manipuladas, não ativaram os pontos auriculares e que foram colocadas em pontos não importantes para o parto - ou que não receberam nenhuma intervenção. Os principais achados foram a redução tanto nos escores de avaliação da percepção da dor quanto no padrão de piora progressiva dela em até 120 minutos, assim como um menor uso de fármacos analgésicos.

Em outro estudo, Mafetoni RR e Shimo AKK (2016a), demonstraram resultados semelhantes na eficácia do controle da dor, contudo, foi evidenciado que a auriculoterapia ainda é desconhecida pela maioria das parturientes, apesar de ser uma técnica que tem mostrado bons resultados. 
Já o outro método da medicina tradicional chinesa é a acupressão. Essa terapia tem o objetivo de manter o equilíbrio das energias corporais a partir da realização de pressão sobre determinadas estruturas corporais, resultando em redução da dor e sensação de relaxamento (MAFETONI RR e SHIMO AKK, 2016b). Os principais pontos citados pelos artigos foram o Spleen 6 (SP6) e o Large Intestinal 4 (LI4) (TÜRKMEN H e TURFAN, 2019; YILDIRIM E, et al., 2018; HAMLACđ Y e YAZICI S, 2017; OZGOLI G et al., 2016).

Os resultados encontrados em todos eles foram redução da dor no parto, diminuição da duração média do primeiro estágio dele e aumento na sensação de satisfação da parturiente. Em ambos artigos, esses efeitos foram observados em pacientes submetidas a acupressão durante a fase ativa do trabalho de parto e quando comparadas a outras mulheres que receberam apenas o tratamento convencional de cada maternidade (TÜRKMEN H e TURFAN, 2019; YILDIRIM E, et al., 2018; HAMLACđ Y e YAZICI S, 2017; MAFETONI RR e SHIMO AKK, 2016, OZGOLI G, et al., 2016).

Vale destacar que, ao associar a acupressão ao uso de bolsas de gelo, os desfechos esperados foram os mesmos nas gestantes que foram submetidas ao tratamento completo - acupressão associado à bolsa de gelo - em relação às que receberam apenas aplicação de gelo no mesmo ponto e às que receberam o protocolo padrão da maternidade (YILDIRIM E, et al., 2018).

Além disso, há métodos não farmacológicos que consistem no calor aplicado à parturiente. Um exemplo é a hidroterapia, que é um jato de água direcionado à região lombo-sacral da mulher, por um período de tempo determinado, com a temperatura de aproximadamente $37^{\circ} \mathrm{C}$. Ela pode estar em pé ou sentada, conforme sua preferência.

De acordo com Cavalcanti ACV, et al. (2019), essa terapia causa estimulação cutânea capaz de reduzir os níveis de hormônios neuroendócrinos relacionados ao estresse e a ansiedade, regular o padrão das contrações uterinas, causando relaxamento e, consequentemente, bem estar. Estes mecanismos também ocorrem ao colocar uma bolsa de água quente na região sacro-perineal da gestante, que de acordo com Taavoni S, et al. (2016), é capaz de reduzir a dor durante o trabalho de parto.

Cavalcanti ACV, et al. (2019) compara o uso da hidroterapia e da bola suíça e, também, o uso destas combinadas. A bola suíça estimula a posição vertical da mulher, auxiliando na descida e apresentação fetal, além de causar relaxamento da musculatura lombar e do assoalho pélvico, aliviando o desconforto. O seu modo de uso é com a parturiente sentada sobre a bola, com a perna flexionada formando um ângulo de $90^{\circ}$ e a região plantar dos pés apoiada no chão, enquanto realiza movimentos de propulsão e rotação pélvica.

Foi verificado que as terapias analisadas não interferem na redução da dor durante o trabalho de parto, entretanto, ambas abreviaram o tempo deste, principalmente ao serem utilizadas em conjunto. Ao analisar o uso da bola suíça e o da hidroterapia separadamente, a hidroterapia foi mais efetiva, por apresentar maior frequência de contrações uterinas e dilatação cervical, resultando assim em um menor tempo de duração do trabalho de parto.

De acordo com Akkoz S e Karaduman S (2019), a massagem na região sacral melhora a tolerância da dor da parturiente, além de aumentar o seu vínculo com a enfermeira que está realizando-a, contribuindo assim para a coragem e a participação da mulher, que relata uma maior satisfação após a concepção ao realizar esta prática. A massagem, apesar de não reduzir o tempo do parto, faz com que a fadiga e a ansiedade diminuam, os espasmos musculares relaxem e a atenção seja distraída. Além de todos os seus benefícios, ela é o método de estimulação tátil mais antigo usado para aliviar a dor do parto. Ela é simples, acessível, segura e muito aceitável pelas mulheres grávidas (SURUCU SG, et al., 2018).

Já o uso da música também é uma terapia de relaxamento, que desvia a atenção da dor e reduz a ansiedade da mulher. Isso acontece por causa do aumento da secreção de endorfina que ocorre ao escutála (ERDORGAN SU, et al., 2017).

A prática de Yoga antecedendo o trabalho de parto pode, também, impactar positivamente na experiência das parturientes. Segundo Jadhi F, et al. (2017), a realização de exercícios de yoga três vezes por semana durante pelo menos uma hora, entre a $26^{\circ}$ e a $37^{\circ}$ semana de gestação, além de reduzir os escores de dor em comparação às mulheres que não fizeram essa prática, reduziu a duração média do segundo e terceiro estágios e a taxa de necessidade de cesarianas. 
O benefício da redução do escore de dor com a prática de yoga pode ser relacionado à maior circulação de líquido cefalorraquidiano e sua relação com uma maior disponibilidade de endorfinas e serotonina circulantes, que por sua vez exercem impacto importante no limiar doloroso. Outros benefícios conhecidos dessa prática milenar incluem a melhora da postura materna, o fortalecimento das musculaturas lombar, abdominal e pélvica, o aumento da propriocepção interna, a melhora da respiração, principalmente o desenvolvimento da respiração diafragmática, além do aumento de energia e relaxamento materno.

O controle da respiração é um dos métodos mais conhecidos para esse momento do parto. Acredita-se que os exercícios respiratórios, ao aumentarem a quantidade de oxigênio disponível para a mãe e para o feto, aumentam o relaxamento e a confiança, deixando a mulher mais calma e satisfeita, além de reduzir o desconforto e a dor. O estudo realizado por Yuksel H, et al. (2017) demonstrou que os exercícios de respiração durante o segundo estágio do parto são eficazes no alívio da dor, ajudam na descida do feto e reduzem a pressão exercida no períneo.

Quanto às repercussões fetais, não foram identificadas alterações no Apgar score do feto entre o grupo experimental e o grupo controle. Quando realizados na fase ativa do primeiro estágio do trabalho de parto, de acordo com Boaviagem A, et al. (2017), os exercícios respiratórios não se mostraram eficazes no controle da dor, da ansiedade, da fadiga e no aumento da satisfação materna. Dessa maneira, não fica claro se as contradições encontradas estão relacionadas ao estágio do trabalho de parto no qual o método foi aplicado ou se levantam questionamentos acerca da real eficácia da respiração como tratamento não-farmacológico para o alívio da dor. Fica evidente a necessidade de mais estudos de larga escala e de alta qualidade para averiguar a eficácia e os reais benefícios do método de exercícios respiratórios para o manejo da dor durante o trabalho de parto.

\section{CONSIDERAÇÕES FINAIS}

A utilização das terapias complementares discutidas anteriormente são importantes aliadas no controle da dor e, juntamente com o acompanhamento psicológico adequado, com a presença do acompanhante e principalmente com o auxílio de um grupo multidisciplinar com doulas, fisioterapeutas e enfermeiros pode promover uma sensação de satisfação e fornecer um suporte para as parturientes. Portanto, os métodos não farmacológicos são ferramentas simples e que podem acarretar uma melhor vivência do trabalho de parto, viabilizando um parto mais humanizado ao colocar a mulher como protagonista.

\section{REFERÊNCIAS}

1. AKKÖZ ÇS, KARADUMAN S. The effect of sacral massage on labor pain and anxiety: A randomized controlled trial. Japan Journal of Nursing Science, 2020; 17(1): 1-9.

2. BOAVIAGEM A, et al. The effectiveness of breathing patterns to control maternal anxiety during the first period of labor: A randomized controlled clinical trial. Complementary Therapies in Clinical Practice, 2017; 26: 30-35.

3. CAVALCANTI ACV, et al. Terapias complementares no trabalho de parto: ensaio clínico randomizado. Revista Gaúcha de Enfermagem, Porto Alegre, 2019; 40: 1-9.

4. ERDOGAN SU, et al. Effects of low back massage on perceived birth pain and satisfaction. Complementary Therapies in Clinical Practice, 2017; 28: 169-175.

5. HAMDAMIAN S, et al. Effects of aromatherapy with Rosa damascena on nulliparous women's pain and anxiety of labor during first stage of labor. Journal of Integrative Medicine, 2018; 16(2): 120-125.

6. HAMLACđ Y, YAZICI S. The Effect of Acupressure Applied to Point LI4 on Perceived Labor Pains. Holistic Nursing Practice, 2017; 31(3): 167-176.

7. JAHDI F, et al. Yoga during pregnancy: The effects on labor pain and delivery outcomes (A randomized controlled trial). Complementary Therapies in Clinical Practice, 2017; $27: 1-4$.

8. LARA SRG, et al. Vivência de mulheres em trabalho de parto com o uso de essências florais. Rev Fun Care Online, 2020; 12:162-168.

9. MAFETONI RR, et al. Effectiveness of auricular therapy on labor pain: a randomized clinical trial. Texto \& Contexto Enfermagem, 2019; 28: 1-14.

10. MAFETONI RR, SHIMO AKK. Efeitos da auriculoterapia sobre a dor do trabalho de parto: ensaio clínico randomizado. Revista da Escola de Enfermagem da USP., 2016a; 50(5): 726-732.

11. MAFETONI RR, SHIMO AKK. The effects of acupressure on labor pains during child birth: randomized clinical trial. Revista Latino-americana de Enfermagem, 2016b; 24: 1-8.

12. OZGOLI G, et al. Effect of LI4 and BL32 acupressure on labor pain and delivery outcome in the first stage of labor in primiparous women: a randomized controlled trial. Complementary Therapies In Medicine, 2016; 29: 175-180. 
13. SILVA, CA, LARA SRG. Uso do banho de aspersão associado à bola suíça como método de alívio da dor na fase ativa do trabalho de parto. BrJP, 2018; 1(2), 167-170.

14. SURUCU SG, et al. The effect of music on pain and anxiety of women during labour on first time pregnancy: A study from Turkey. Complementary Therapies in Clinical Practice, 2018; 30: 96-102.

15. TAAVONI S, et al. Birth ball or heat therapy? A randomized controlled trial to compare the effectiveness of birth ball usage with sacrum-perineal heat therapy in labor pain management. Complementary Therapies in Clinical Practice, 2016; 24: 99-102.

16. TANVISUT R, et al. Efficacy of aromatherapy for reducing pain during labor: a randomized controlled trial. Archives of Gynecology and Obstetrics, 2018; 297(5): 1145-1150.

17. TÜRKMEN H, TURFAN EÇ. The effect of acupressure on labor pain and the duration of labor when applied to the SP6 point: Randomized clinical trial. Japan Journal Of Nursing Science, 2019; 17(1): 1-9.

18. YAZDKHASTI M, PIRAK A. The effect of aromatherapy with lavender essence on severity of labor pain and duration of labor in primiparous women. Complementary Therapies in Clinical Practice, 2018; 25: 81-86.

19. YILDIRIM E, et al. The effect of ice pressure applied on large intestinal 4 on the labor pain and labor process. Complementary Therapies In Clinical Practice, 2018, 32:25-31.

20. YUKSEL $\mathrm{H}$, et al. Effectiveness of breathing exercises during the second stage of labor on labor pain and duration: a randomized controlled trial. Journal of Integrative Medicine, 2017, 15:456-461. 